\title{
Detection of Incipient Clogging in Pulverized Coal Injection Lines
}

\author{
Andreas Johansson and Alexander Medvedev \\ e-Mail Andreas.Johansson@sm.luth.se, Alexander.Medvedev@sm.luth.se \\ Phone +46920723 34, +46920913 02, Fax +46920915 58 \\ Luleå University of Technology, SE-971 87 Luleå, Sweden
}

\begin{abstract}
This paper deals with the detection of clogging in the coal injection lines of a blast furnace. A physical model is developed and augmented with a signal that represents clogging. An observer is proposed and shown to have any predeflned convergence rate. An adaptive detection threshold scheme which is robust against time-varying uncertainties is employed. Simulations and experiments with real data are carried out to illustrate the usefulness of the methods.
\end{abstract}

\section{INTRODUCTION}

The injection of coal powder is an important tool for reducing the costs and increasing the controllability of the blast furnace process. Coal powder is less expensive than coke and it is injected directly into the active zone of the blast furnace in contrast to coke that has a delay of several hours before reaching this region.

Prerequisites for taking advantage of these benefits are that the coal injection plant is reliable and distributes the coal powder evenly around the blast furnace. Clogging of the individual injection lines conveying the coal powder is a problem that often arises in this context. See also [1] for a design approach and a successful implementation of an improved control system for a coal injection plant.

Early stages of clogging can be dealt with by purging the line with nitrogen or even by sending a pulse to a control valve. Severe cases may, on the other hand, demand that the line has to be disassembled and cleared manually. Thus, in order to uphold even distribution of coal powder and to avoid maintenance stops, preventive actions must be taken at an early stage of clogging. However, to avoind extensive wear of the equipment, this must be done only when necessary.

Furthermore, the complicated relation between the pressures and control signals on the one hand and the coal powder flow on the other hand calls for an automatic detection method. These facts motivate the use of fault detection techniques for detecting clogging as early as possible.

This paper describes modeling of the injection process and the use of fault detection to determine if a clogging is building up. Successful model-based methods for detecting gas leakages have been developed before [2], [3]. A leakage, which is caused by a hole, and a clogging, which can be regarded as an orifice, can both be described by valve models.

There exist several approaches to fault detection. For a recent survey see [4]. Common for many of the model- based fault detection techniques is the use of an observer. The purpose of the observer is to compare the nominal behaviour of the plant to the actual measured signals and generate a residual as the difference between those. The residual, which contains information about faults, can then be analyzed directly, by e.g. some stochastic or deterministic approach. Otherwise, the observer can be augmented by an extra state variable that represents the fault and the residual is used as input when estimating this signal.

In this paper, the latter approach is used and the fault is seen as a bias to be estimated. Reference [5] is a recent paper on the use of the separate-bias technique with numerous references to other articles on this subject. See also [6] for a survey on various applications of fault detection.

The coal injection plant described in this paper is installed at LKAB's experimental blast furnace at the Foundation for Metallurgical Research, MEFOS [7], in Luleå, Sweden.

\section{Modeling}

The experimental blast furnace at MEFOS has three injection lines, each one equipped with flow and pressure sensors and a flow control valve (Fig. 1).

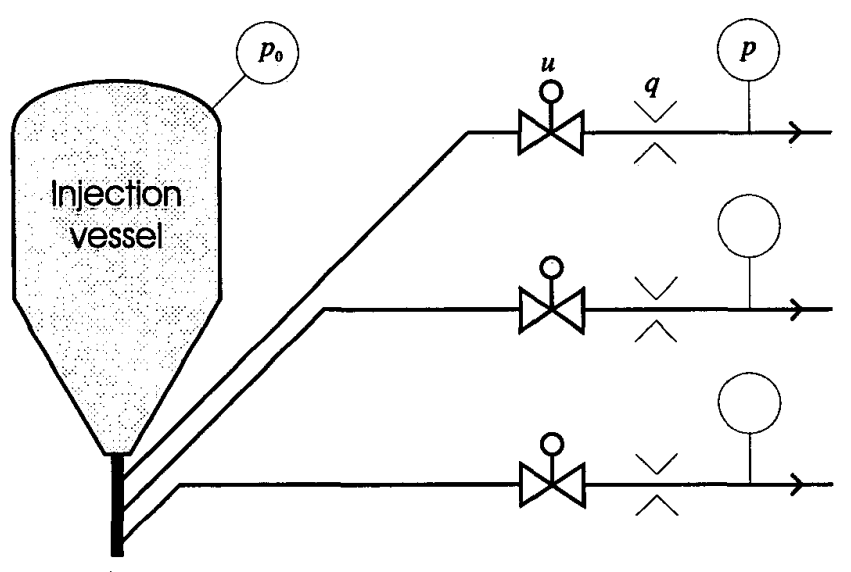

Fig. 1. Overview of the injection system

A pressure sensor is also installed on the injection vessel from which the flow emerges. The symbols in the figures are explained in Table I.

A clogging is regarded as a restriction with unknown area $a_{f}$. In the nominal model (no clogging), the entire 


\begin{tabular}{|l|l|}
\hline Symbol & Meaning \\
\hline \hline$\rightarrow-$ & $\begin{array}{l}\text { Coal line with indicated flow di- } \\
\text { rection }\end{array}$ \\
\hline
\end{tabular}

TABLE I

EXPLANATION OF SYMBOLS

pressure drop $p_{0}-p$ is assumed to take place in the control valve and none in the piping (or clogging). Thus, the faultfree case must be equivalent to clogging with infinite area. This is of course not very accurate when $a_{f}$ is large. In the analysis, however, the inverse of $a_{f}$ will be used instead, which will be small in the case of no clogging.

The location of a clogging can principally be divided into two categories. Fig. 2 shows the first case when the clogging, represented as an extra valve, is located after the pressure measurement. In this case, the clogging detection

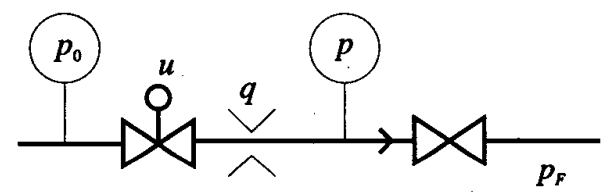

Fig. 2. Clogging after the pressure measurement

comes down to detecting a nonzero pressure drop $p-p_{F}$ where $p_{F}$ is the pressure in the furnace. This pressure is not measured, but it can be approximated by e.g. the minimum or the mean of the pressures of the other injection lines, if these are assumed not to be clogged. Due to measurement noise and the error in the approximation of $p_{F}$, the pressure drop $p-p_{F}$ is almost always nonzero, even when there is no clogging, Thus some measures must be taken in order to avoid false alarms. Low-pass filtering of the pressure drop reduces the effect of measurement noise and a detection threshold that accounts for the possible error in $p_{F}$ must be included.

Fig. 2 shows the second case, where the clogging is located before the pressure measurement. It can also be located before the control valve, but this makes no difference in the analysis.

Since the coal powder is rather dense in the injection lines, it is regarded as a liquid. The flow of liquid through

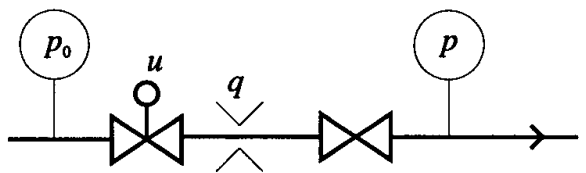

Fig. 3. Clogging before the pressure measurement

a restriction can be calculated as

$$
q=a \sqrt{2 \rho \Delta p} \triangleq a g(\Delta p)
$$

where $\Delta p$ is the pressure drop over the restriction, $\rho$ is the density of the fluid and $a$ represents the minimum crosssection area of the restriction.

Analysis of the system in Fig. 3 involves determining the flow through restrictions connected in series. Fig. 4 shows the general situation with $N$ restrictions. The intermediate pressures $p_{i}, i=0,1, \ldots, N$ and the cross-section areas $a_{i}, i=1,2, \ldots, N$ are indicated.

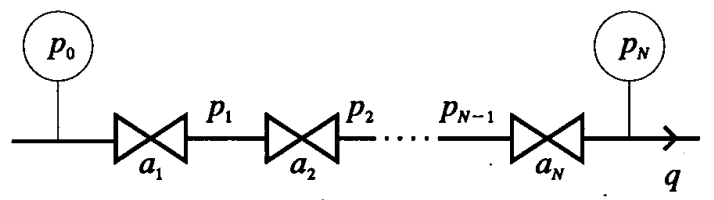

Fig. 4. Restrictions connected in series

The flow $q$ can be calculated using (1) applied to the $i$ :th restriction as

$$
q=a_{i} \sqrt{2 \rho\left(p_{i-1}-p_{i}\right)}
$$

Multiplying the above expression by $a_{i}^{-1}$ and taking the square yields

$$
a_{i}^{-2} q^{2}=2 \rho\left(p_{i-1}-p_{i}\right)
$$

Finally, summing over all $i=1,2, \ldots, N$ and isolating the flow $q$ gives

$$
\begin{aligned}
q & =\left(\sum_{i=1}^{N} a_{i}^{-2}\right)^{-1 / 2} \cdot \sqrt{2 \rho\left(p_{0}-p_{N}\right)} \\
& =\left(\sum_{i=1}^{N} a_{i}^{-2}\right)^{-1 / 2} g\left(p_{0}-p_{N}\right)
\end{aligned}
$$

Applying (2) to the situation in Fig. 3 yields

$$
q=\left(a^{-2}+a_{f}^{-2}\right)^{-1 / 2} g(\Delta p)
$$

where $\Delta p=p_{0}-p$.

The area of the restriction in the valve is assumed to be proportional to the control signal, thus $a=k u$. Since $a_{f}=\infty$ is equivalent to no clogging, defining the fault signal as $f=a_{f}^{-2}$ then gives the desired property that 
clogging is equivalent to $f>0$. With these definitions, the flow can be expressed as

$$
q=\left((k u)^{-2}+f\right)^{-1 / 2} g(\Delta p)
$$

Under normal operation, the fault signal is small, and it is therefore motivated to linearize the system around $f=$ 0 . This is done by taking the derivative of the flow with respect to the fault signal. For small $f=\delta f$, the flow $q$ can thus be approximated by

$$
q=k u g(\Delta p)+\frac{\partial q}{\partial f} \delta f
$$

where the derivative $\partial q / \partial f$ is

$$
\frac{\partial q}{\partial f}=-\frac{k^{3}}{2} u^{3} g(\Delta p) \triangleq q_{f}^{\prime}(u, \Delta p)
$$

Since $u$ and $\Delta p$ are time varying, the functions $g(\Delta p)$ and $q_{f}^{\prime}(u, \Delta p)$ will be replaced by $g(t)$ and $q_{f}^{\prime}(t)$ for convenience.

\section{A. Identification}

The only unknown parameter in the process model (3) is the proportional constant of the valve characteristic $k$, which can be estimated through identification. Fig. 5 shows a simulation of a model where $k$ is estimated from logged data by a least squares criterion.

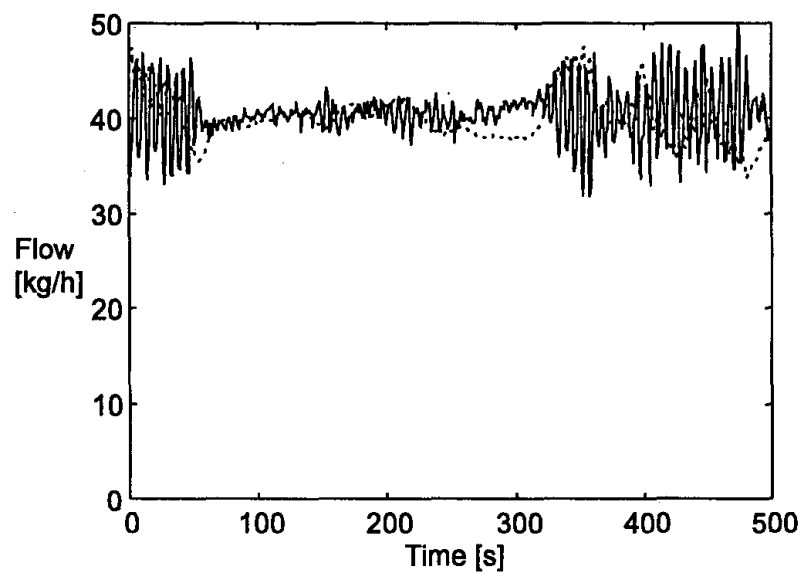

Fig. 5. Simulated flow (solid line) versus measured flow (Dashed line)

From the figure, it is evident that although the level of the flow is predicted very well, the fast oscillations in the simulated signal are not so prominent in the measured signal. The oscillations, that are due to a poorly tuned controller are obviously damped by some dynamics in e.g. the flow control valve.

\section{Clogging Detection}

It is assumed that a clogging develops slowly, and therefore the fault model $\dot{f}=0$ is accepted. The process model can thus be expressed as

$$
\begin{aligned}
\dot{f} & =0 \\
y & =k u(t) g(t)+q_{f}^{\prime}(t) f
\end{aligned}
$$

where $y=q$ is the output signal. The following observer is proposed for the system above

$$
\begin{aligned}
\dot{\hat{f}} & =\frac{K}{q_{f}^{\prime}(t)}(y-\hat{q}) \\
\hat{q} & =k u(t) g(t)+q_{f}^{\prime}(t) \hat{f}
\end{aligned}
$$

where $K$ is a parameter to choose. The estimation error is defined as $\varepsilon \triangleq f-\hat{f}$. A differential equation for $\varepsilon$ is obtained by calculating the derivative.

$$
\begin{aligned}
\dot{\varepsilon} & =\dot{f}-\dot{\hat{f}}=\frac{-K}{q_{f}^{\prime}(t)}(y-\hat{q}) \\
& =\frac{-K}{q_{f}^{\prime}(t)}\left(u(t) g(t)+q_{f}^{\prime}(t) f-u(t) g(t)-q_{f}^{\prime}(t) \hat{f}\right) \\
& =-K(f-\hat{f})=-K \varepsilon
\end{aligned}
$$

The estimation error can thus be made to converge to zero arbitrarily fast by choosing the parameter $K>0$ sufficiently large.

\section{A. Sensitivity analysis}

The measurement of the mass flow $q$ is done indirectly by multiplying the measured velocity of the coal powder by an estimated density. This makes the flow measurement rather uncertain, which fact motivates the introduction of a time-varying, multiplicative uncertainty, $\Delta_{q}(t)$ i.e.

$$
y(t)=q(t)\left(1+\Delta_{q}(t)\right)
$$

Furthermore, due to nonlinear characteristic of the control valve, an uncertainty factor $\Delta_{k}(t)$ is introduced in the expression for the cross-section area $a$, thus

$$
a(t)=u(t) k\left(1+\Delta_{k}(t)\right)
$$

In the nominal plant, the fault signal is zero, which under the uncertainties above gives the measurement signal

$$
y(t)=\left(1+\Delta_{q}(t)\right)\left(1+\Delta_{k}(t)\right) k u(t) g(t)
$$

The uncertainties can be represented by one parameter

$$
\Delta(t)=\Delta_{q}(t)+\Delta_{k}(t)+\Delta_{q}(t) \Delta_{k}(t)
$$

which is limited by $|\Delta(t)|<\delta$. Using $\Delta$ as uncertainty parameter, the measurement signal can be expressed as

$$
y=(1+\Delta(t)) k u(t) g(t)
$$


When uncertainties are present, the estimation error $\varepsilon$ can thus be calculated by

$$
\begin{aligned}
\dot{\varepsilon} & =\dot{f}-\dot{\hat{f}}=\frac{-K}{q_{f}^{\prime}(t)}(y-\hat{q}) \\
& =\frac{-K}{q_{f}^{\prime}(t)}\left((1+\Delta(t)) k u(t) g(t)-k u(t) g(t)-q_{f}^{\prime}(t) \hat{f}\right) \\
& =-K \frac{\Delta(t) k u(t) g(t)}{q_{f}^{\prime}(t)}-K \varepsilon=\frac{2 K \Delta(t)}{k^{2} u(t)^{2}}-K \varepsilon
\end{aligned}
$$

\section{B. Modified observer}

Since the fault signal $f$ can never be negative, the observer can be modified to

$$
\begin{aligned}
& \dot{\hat{f}}= \begin{cases}0 & (\hat{f}=0) \wedge\left(\hat{f}_{d} \leq 0\right) \\
\hat{f}_{d} & \text { Otherwise }\end{cases} \\
& \hat{q}=k u(t) g(t)+q_{f}^{\prime}(t) \hat{f}
\end{aligned}
$$

where

$$
\hat{f}_{d}=\frac{K}{q_{f}^{\prime}(t)}(y-\hat{q})
$$

The above modification to the observer ensures that the fault estimation does not go below zero.

\section{THRESHOLD SELECTION}

Since the estimated fault $\hat{f}$ will be nonzero almost all the time, due to measurement noise and model uncertainties, a threshold $\varphi$ is needed, Clogging is thus said to have taken place if $\hat{f}>\varphi$. To avoid false alarms, the threshold should be designed so that $\hat{f}<\varphi$ provided that there is no clogging and the uncertainties are inside the specified bounds. The threshold should thus satisfy

$$
\varphi(t)=\sup _{|\Delta|<\delta, f=0} \hat{f}(t)
$$

An upper bound for the fault estimate $\hat{f}$ in the case of no fault must therefore be calculated. But since $\varepsilon=-\hat{f}$ when $f=0$, the error equation (4) with $\varepsilon$ substituted by $-\hat{f}$ can be used. The clogging estimate $\hat{f}$ is thus determined by the differential equation

$$
\dot{\hat{f}}+K \hat{f} \equiv h(t)
$$

where

$$
h(t)=-\frac{2 K \Delta(t)}{k^{2} u(t)^{2}}
$$

The differential equation (6) is linear and time-invariant and has the solution.

$$
\hat{f}=e^{-K t}\left(\int e^{K t} h(t) d t+C\right)
$$

where the constant $C$ must be determined by some initial condition. If it is assumed that the observer is initialized to zero i.e. $\hat{f}(0)=0$ then the solution reduces to

$$
\begin{aligned}
\hat{f}(t) & =e^{-K t} \int_{0}^{t} e^{K \tau} h(\tau) d \tau \\
& =-\frac{2 K}{k^{2}} e^{-K t} \int_{0}^{t} \frac{\Delta(\tau) e^{K \tau}}{u(\tau)^{2}} d \tau
\end{aligned}
$$

The threshold is determined by calculating an upper bound for the estimated fault signal $\hat{f}$

$$
\begin{aligned}
|\hat{f}| & \leq\left|-\frac{2 K}{k^{2}} e^{-K t} \int_{0}^{t} \frac{\Delta(\tau) e^{K \tau}}{u(\tau)^{2}} d \tau\right| \\
& \leq \frac{2 K}{k^{2}} e^{-K t} \int_{0}^{t} \frac{|\Delta(\tau)| e^{K \tau}}{u(\tau)^{2}} d \tau \\
& \leq \frac{2 K \delta}{k^{2}} e^{-K t} \int_{0}^{t} \frac{e^{K \tau}}{u(\tau)^{2}} d \tau
\end{aligned}
$$

The last expression is defined to be the threshold

$$
\varphi(t)=\frac{2 K \delta}{k^{2}} e^{-K t} \int_{0}^{t} \frac{e^{K \tau}}{u(\tau)^{2}} d \tau
$$

The expression (7)-is however not very practical to use numerically, since the growing integral is to be multiplied by a decreasing exponential function. In order to obtain a differential equation instead, the time derivative of the threshold is calculated as

$$
\dot{\varphi}(t)=\frac{2 K \delta}{k^{2}}\left(-K e^{-K t} \int_{0}^{t} \frac{e^{K \tau}}{u(\tau)^{2}} d \tau+\frac{1}{u(t)^{2}}\right)
$$

which can be simplified to give the differential equation

$$
\dot{\varphi}(t)=-K \varphi(t)+\frac{2 K \delta}{k^{2} u(t)^{2}}
$$

\section{A. Modified threshold}

If the modified observer (5) is used, then the following equation for calculating the threshold can be applied.

$$
\dot{\varphi}(t)= \begin{cases}-\bar{K} \varphi(t) & \hat{f}=0 \\ -K \varphi(t)+\frac{2 K \delta}{k^{2} u(t)^{2}} & \text { Otherwise }\end{cases}
$$

The threshold above will converge to zero whenever the clogging estimate is zero.

\section{V, Simulations}

The conditions of the simulations are the following. The control valve parameter is $k=10^{-4} \mathrm{~m}^{2}$, uncertainty bound $\delta=0.1$, pressure drop $\Delta p(t)=70 \mathrm{kPa}$ and valve uncertainty $\Delta_{k}(t)=0$. The control signal is changed from 0.3 to 0,2 at $t=40 \mathrm{~s}$ and the observer parameter $K$ is set to 0.1 . 
In the first example the flow measurement uncertainty $\Delta_{q}$ is changed from 0 to -0.08 at $t=20 \mathrm{~s}$. The clogging area $a_{f}$ is changed from $\infty$ to $0.5 \cdot 10^{-4} \mathrm{~m}^{2}$ at $t=60 \mathrm{~s}$. Fig. 6 shows the real and the estimated fault signal as well as the detection threshold. The fault estimate exceeds the detection threshold in 3.5 seconds after the clogging has occurred at $t=60 \mathrm{~s}$. Fig. 7 shows the measured and the

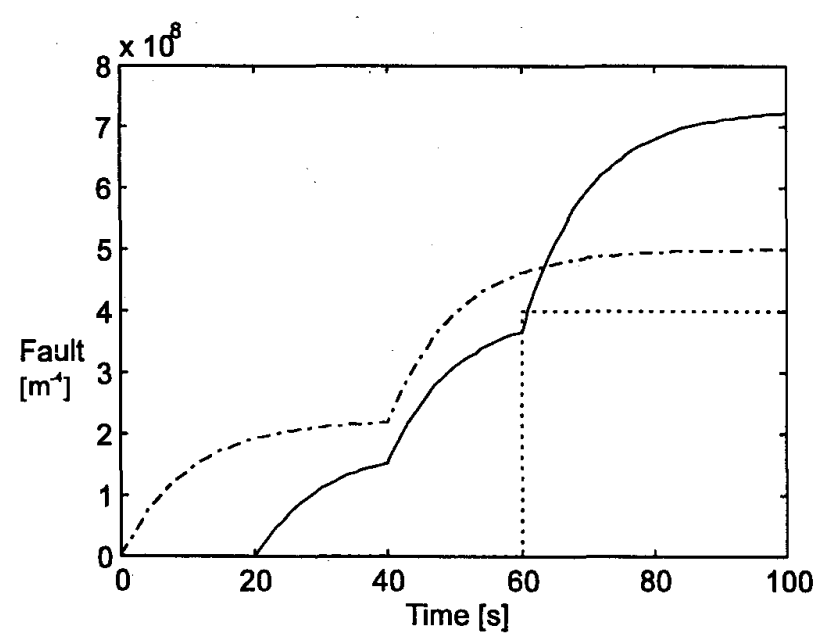

Fig. 6. Example 1. Fault signal (dashed line), fault estimate (solid line) and detection threshold (dash-dotted line)

estimated mass flow. Note the small decrease in flow that the clogging introduces at $t=60 \mathrm{~s}$.

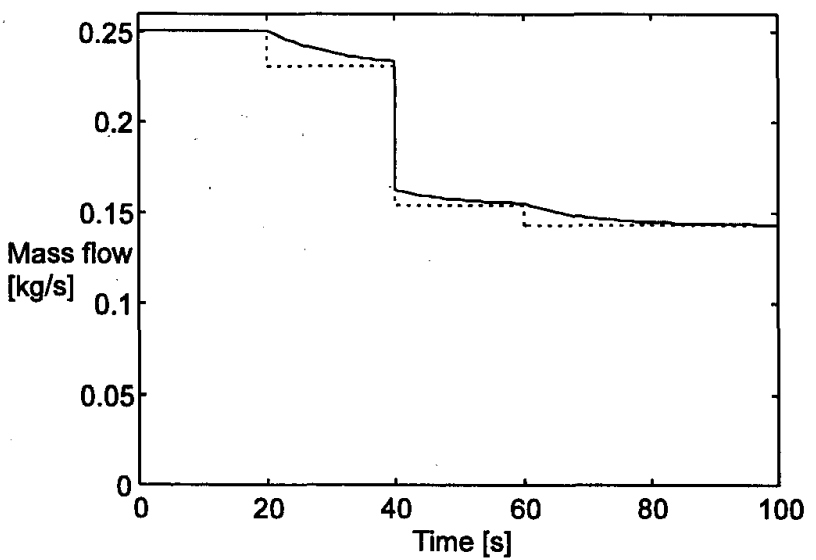

Fig. 7. Example 1. Flow measurement (dashed line) and flow estimate (solid line)

In the second example, a sequence of uniformly distributed random numbers between -0.1 and 0.1 with sampling time 1 second is used as the measurement uncertainty. The clogging area is somewhat smaller, $a_{f}=$ $0.3 \cdot 10^{-4} \mathrm{~m}^{2}$. Fig. 8 and Fig. 9 show the fault signals and the mass flow signals, respectively. Detection of the

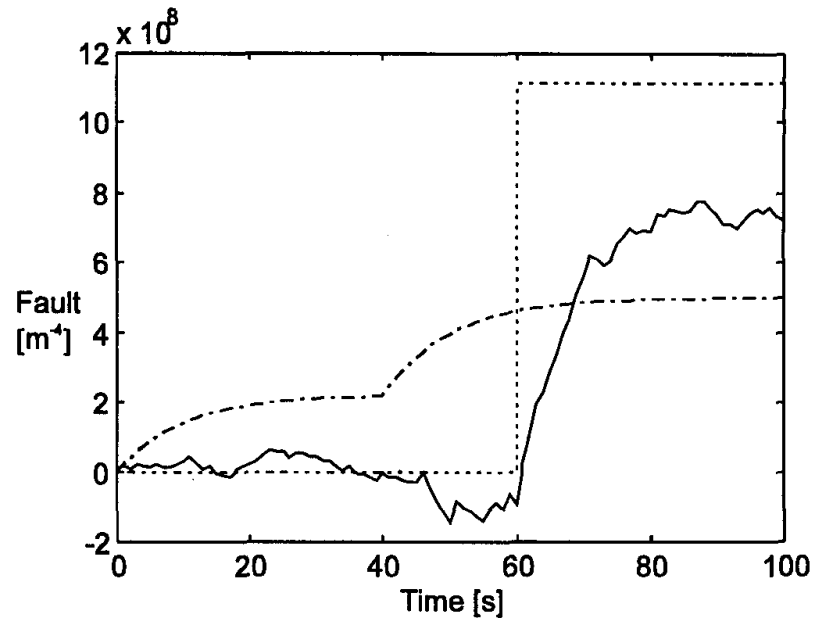

Fig. 8. Example 2. Fault signal (dashed line), fault estimate (solid line) and detection threshold (dash-dotted line)

clogging is done within 9 seconds after it has taken place.

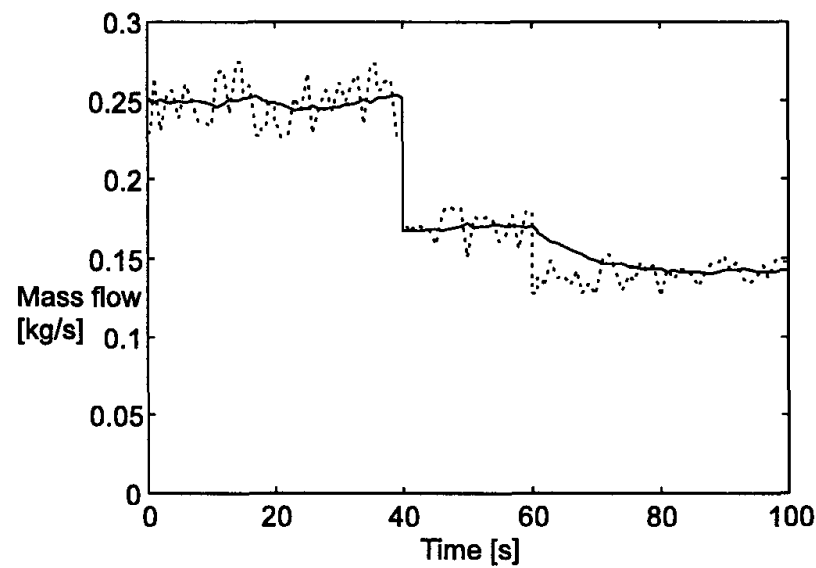

Fig. 9. Example 2. Flow measurement (dashed line) and flow estimate (solid line)

In the third example, the modified observer and threshold are used. Fig. 10 and Fig. 11 show the fault signals and the mass flow signals, respectively. The clogging is detected in 6 seconds after it as has occurred.

From the figures above it is evident that the estimate of the fault signal $f$ is not very accurate. There are two reasons for this.

1. There are uncertainties in the valve characteristic and the flow measurement

2. The process model used in the observer is a linearization of the physical process model and is only valid for $f \approx 0$

The numerical value of the fault signal is, however, not important, since it is only used to determine whether there is clogging or not. 


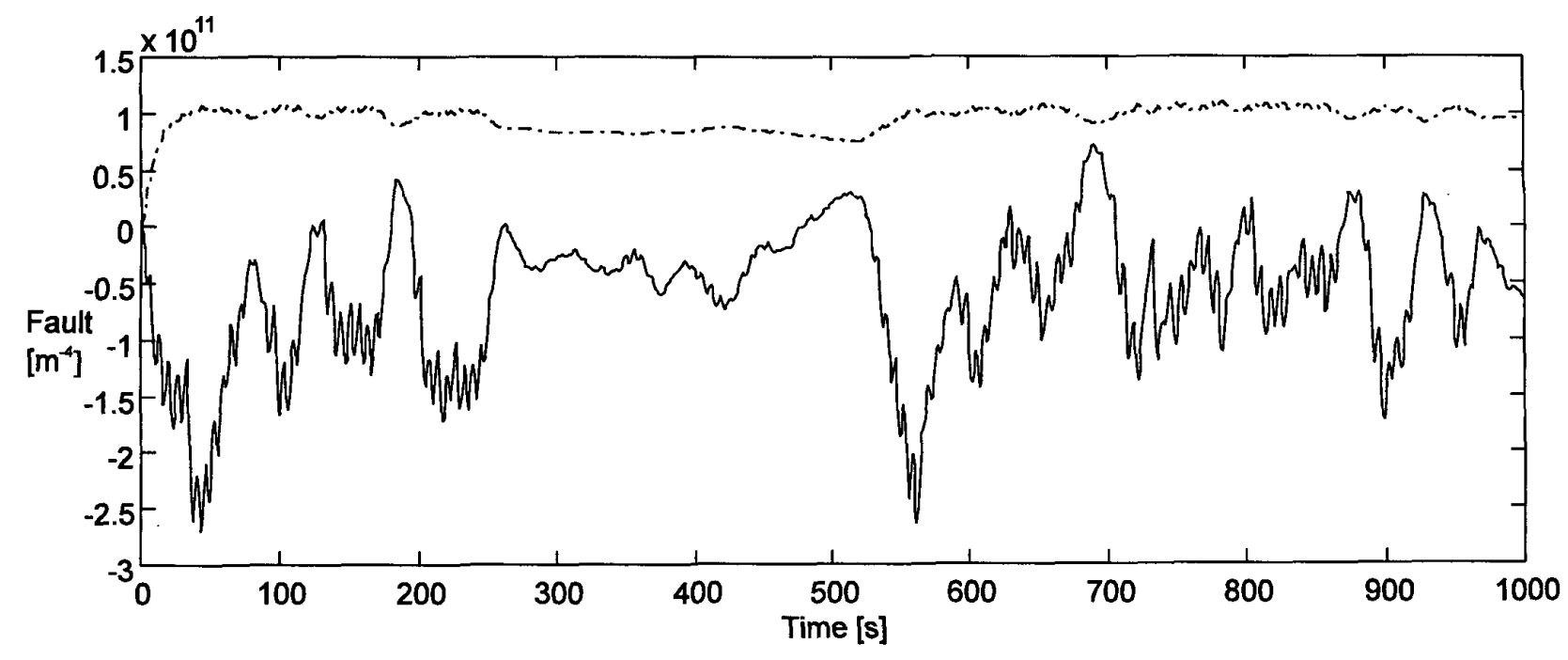

Fig. 12. Simulation with real data. Fault estimate (solid line) and detection threshold (dash-dotted line)

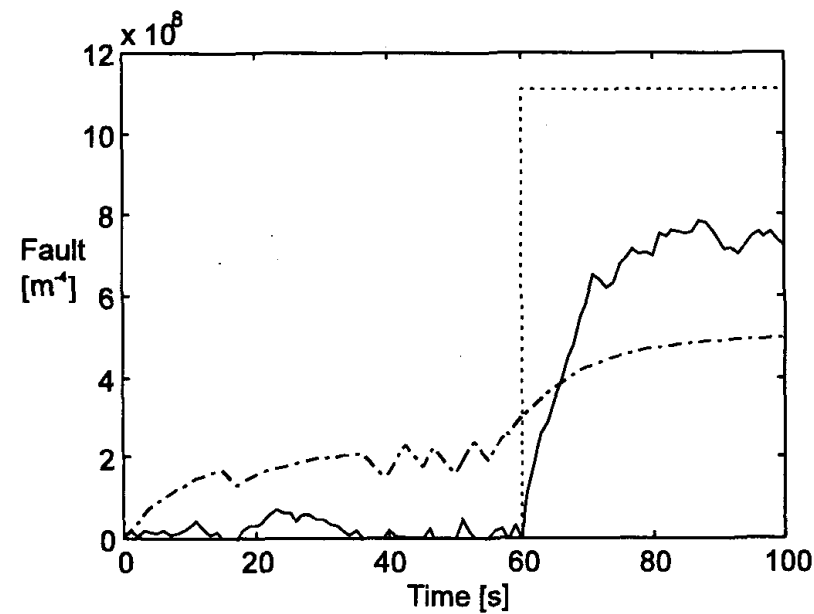

Fig. 10. Example 3. Fault signal (dashed line), fault estimate (solid line) and detection threshold (dash-dotted line)

\section{A. Experiments with real data}

Fig. 12 and Fig. 13 show simulations with measured data. In Fig. 13, the modified observer (5) and threshold (8) is used. The parameters are the same as in the previous simulations i.e. $K=0.1$ and $\delta=0.1$. Note that the fault estimate never exceeds the detection threshold, that is, no false alarm is raised.

\section{FUTURE WORK}

An uncertainty bound $\delta$ can be found by examining the variations in the identified parameter $k$. An optimal value for the observer parameter $K$ could also be calculated, on

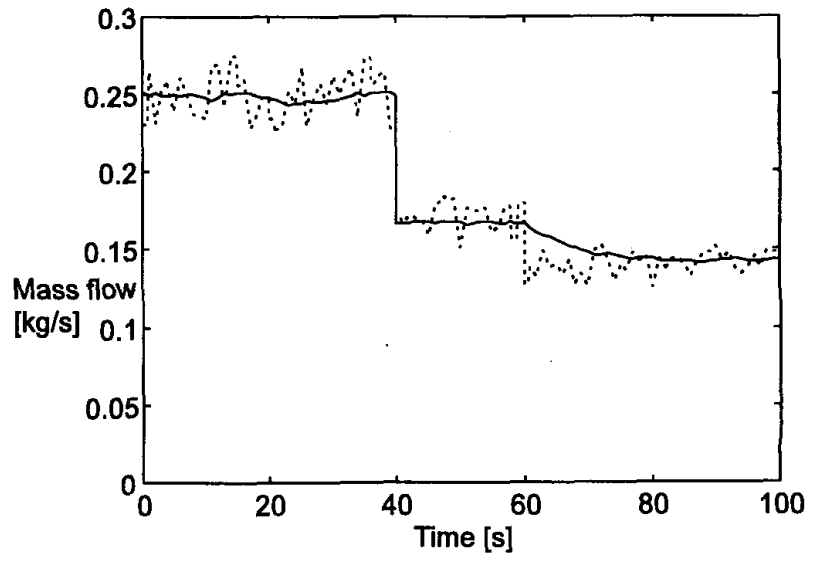

Fig. 11. Example 3. Flow measurement (dashed line) and flow estimate (solid line)

the basis of the properties of the measurement noise.

Furthermore, an extension of the method for the case when flow measurement is not available will be developed. Preliminary results show that by using the measurement of the weight of the injection vessel, an observer can be constructed that under certain conditions can detect and isolate a clogging in an injection line.

The presented method for clogging detection will be implemented as an option in the next version of SafePCI [1], which is a commercially available system for control and fault detection in pulverized coal injection plants. 


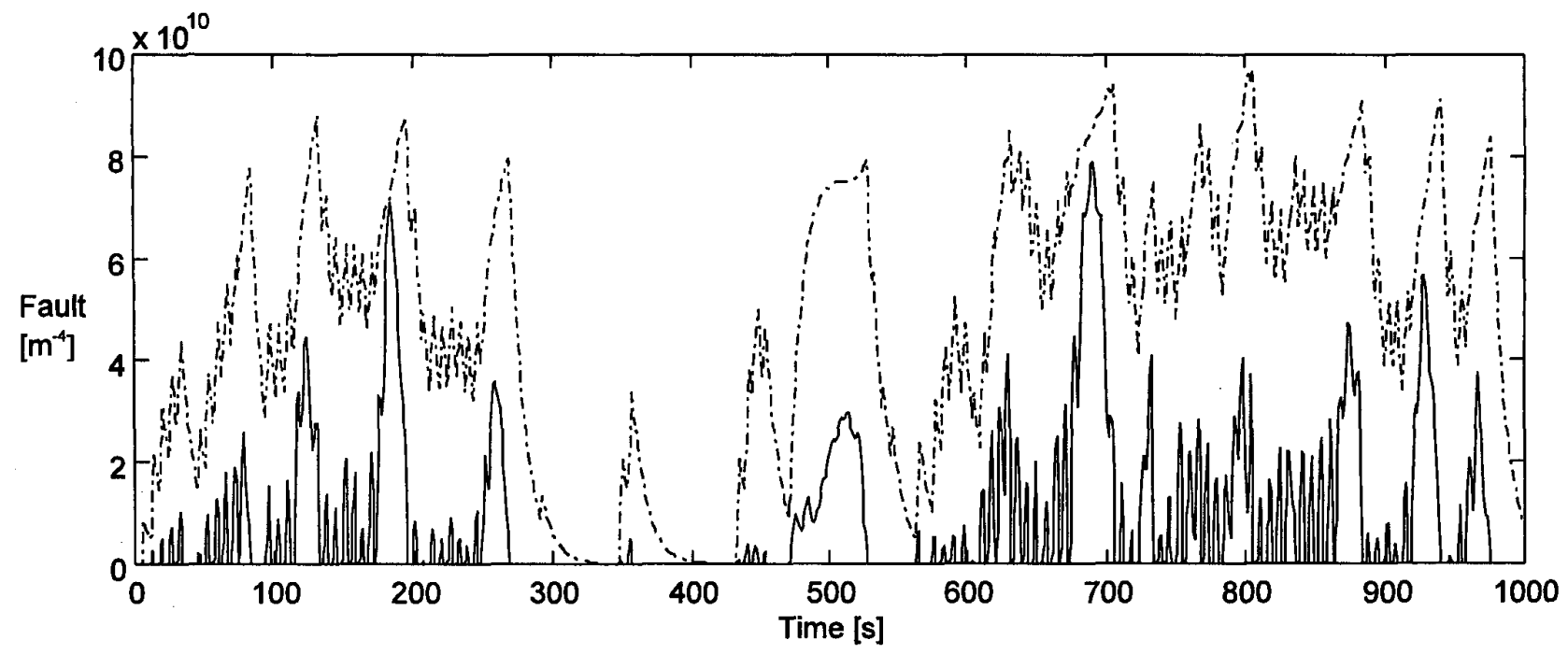

Fig. 13. Simulation with real data using the modified observer and the modified threshold. Fault estimate (solid line) and detection threshold (dash-dotted line)

\section{CONCLUSIONS}

A method for detecting clogging in the coal injection pipes of a blast furnace process was presented. This was done by developing a physical model and a corresponding observer for the injection lines. The observer has a state variable that represents the clogging. An adaptive threshold, which is robust against time-varying uncertainties was also suggested. Simulations and experiments with measured data showed that the method is useful.

\section{ACKNOWLEDGMENT}

Financial support of the Center for Process and System Automation (ProSA) at Luleå University of Technology provided by Norrbottens Forskningsråd is gratefully acknowledged.

\section{REFERENCES}

[1] W. Birk, A. Johansson, and A. Medvedev, "Model-based control for a fine coal injection plant," IEEE Control Systems Magazine, vol. 19, pp. 33-43, February 1999.

[2] A. Johansson and A. Medvedev, "Model based leakage detection in a pulverized coal injection vessel." IEEE Transactions on Control Systems Technology, in press.

[3] A. Johansson, W. Birk, and A. Medvedev, "Model-based gas leakage detection and isolation in a pressurized system via Laguerre spectrum analysis," in Proceedings of the 1998 IEEE International Conference on Control Applications, vol. 1, pp. 212-215, 1998.

[4] P.M.Frank and X.Ding, "Survey of robust residual generation and evaluation in observer-based fault detection systems," J. Proc. Cont., vol. 7, no. 6, pp. 403-424, 1997.

[5] M. Boutayeb, D. Aubry, P. M. Frank, and J. Y. Keller, "A separate-bias observer for non linear discrete-time systems," in IFAC Symposium on Fault Detection, Supervision and Safety for Technical Processes, Kingston-upon-Hull, Hull, UK, 1997.

[6] R. Isermann and P. Ballé, "Trends in the application of model based fault detection and diagnosis of technical processes," in IFAC World Congress Proceedings, 1996. CD-ROM.

[7] http://www.mefos.se/. 